\title{
CRYSTALLITE SIZE DEPENDENCE ON MAGNETOCALORIC EFFECT OF GdAI2 INTERMETALLIC COMPOUND
}

\author{
de Paula, V. G. ${ }^{1}{ }^{*}$; da Silva, L. M. ${ }^{2}$; Coelho, A. A. ${ }^{1}$; dos Santos, A.O. ${ }^{2}$; Cardoso, L.P. ${ }^{1}$ \\ ${ }^{1}$ DFA, IFGW, UNICAMP,Campinas, SP, Brasil \\ ${ }^{2}$ CCSST, UFMA, Imperatriz, MA, Brasil
}

*vinicius.gomes.paula@gmail.com

\begin{abstract}
In this work, the magnetic, magnetocaloric and structural properties of rare earth based $G d A l_{2}$ intermetallic compound on nanometric scale were studied, focusing on the crystallite size dependence of the magnetocaloric effect. The nanoparticles were obtained in a mechanical milling process in which the alloys, previously synthetized in an arc furnace under argon atmosphere, were crushed inside a planetary high energy ball system also in an argon atmosphere at different milling times. The nanoparticles structure were analyzed by X-ray diffraction, as well as lattice strain and crystallite size values. Rietveld refinement method analysis of the experimental data has confirmed the existence of a single cubic phase for the $G_{d A l}$ series, as expected for a Laves phase compound. As the milling time increases, one has observed the crystallite size decreases down to $19 \mathrm{~nm}$ the minimum value. The lattice values increases due to atomic disorder induced by the mechanical milling process. Magnetic properties are also strongly crystallite size dependent, evidenced by the temperature interval enlargement of the second order magnetic transition as the particle size decreases. A table-like behavior with improved RCP (Relative Cooling Power) was obtained for larger crystallite size samples, while smaller crystallite size ones exhibited an increased $\left|-\mathrm{D} S_{M m a ́ x}\right|$ at cryogenic temperatures, associated with the superparamagnetism. Therefore, the magnetocaloric effect on the $\mathrm{GdAl}_{2}$ nanostructured compounds was optimized in comparison to the bulk sample.
\end{abstract}

